\title{
Televisione e terrorimso nel romanzo Nucleo Zero di Luce d'Eramo
}

Daniella Ambrosino

\section{OpenEdition}

Journals

Edizione digitale

URL: http://journals.openedition.org/cei/103

DOI: $10.4000 /$ cei. 103

ISSN: 2260-779X

\section{Editore}

UGA Éditions/Université Grenoble Alpes

\section{Edizione cartacea}

Data di pubblicazione: 15 juin 2010

Paginazione: 53-62

ISBN: 978-2-84310-168-7

ISSN: $1770-9571$

\section{Notizia bibliografica digitale}

Daniella Ambrosino, «Televisione e terrorimso nel romanzo Nucleo Zero di Luce d'Eramo», Cahiers d'études italiennes [Online], 11 | 2010, online dal 15 décembre 2011, consultato il 27 mars 2021. URL: http://journals.openedition.org/cei/103 ; DOl: https://doi.org/10.4000/cei.103 


\title{
TELEVISIONE E TERRORIMSO NEL ROMANZO NUCLEO ZERO DI LUCE D'ERAMO
}

\author{
Daniella Ambrosino \\ Magistrate
}

Luce d'Eramo dava molta attenzione ai mezzi di comunicazione di massa. Da ragazza, aveva fatto esperienza dell'enorme importanza che strumenti come i nuovi media di allora, la radio e i cinegiornali, avevano avuto nel costruire il consenso intorno a fascismo e nazismo, e questa consapevolezza non l'ha più abbandonata nel dopoguerra, dove si è sempre criticamente interessata dell'uso dei media in democrazia, e in particolare del medium principe acceso in tutte le case, la televisione.

Un suo libro poco conosciuto, del gennaio 1974, si intitola Cruciverba politico. Come funziona in Italia la strategia della diversione. $\grave{E}$ un saggio e perciò qui non ne parleremo, se non per ricordare un'esperienza che successivamente influì molto sul lavoro narrativo della d'Eramo. È un'analisi molto accurata di come la televisione e la stampa avessero trattato un caso all'epoca clamoroso, il caso Feltrinelli / Cederna: il miliardario editore di sinistra, simpatizzante per i movimenti extraparlamentari, che secondo la polizia era saltato in aria nel tentativo di minare un traliccio dell'alta tensione; e la giornalista che insieme ad altri, sulla scia del caso Valpreda e dell'irrisolta questione delle stragi di Stato, aveva contestato la ricostruzione della questura, denunciando che l'editore era stato assassinato. Ne nacque un'enorme campagna di stampa contro la Cederna, (che finì sotto processo per "propagazione di notizie false e tendenziose»); nel suo libro Luce d'Eramo riscontrò uno slittamento dal caso Feltrinelli al caso Cederna, con elusione degli interrogativi rimasti aperti sulla morte dell'editore, nel quadro di una più ampia strategia di diversione dell'opinione pubblica.

Nel suo libro Luce d'Eramo sottolineava il ruolo determinante svolto nella vicenda dalla tv, che allora era una televisione esclusivamente di Stato (RAI) con il monopolio assoluto dell'informazione televisiva: le emittenti 
private ancora non esistevano. Il progetto della d'Eramo prevedeva di analizzare giorno per giorno non solo la stampa ma anche i telegiornali sul caso Feltrinelli-Cederna; ma la RAI non consentì alla scrittrice di accedere alla documentazione. In una nota in fondo a quel libro oggi introvabile, dopo aver ringraziato i giornali che, senza eccezioni, le avevano aperto i loro archivi, l'autrice scrive: «La radiotelevisione italiana invece ha rifiutato, con lettera dell'ottobre 1972, di lasciarmi esaminare i testi o le registrazioni dei telegiornali, affermando che non vengono conservati.» Palesemente, una scusa ben poco credibile.

Ciononostante la d'Eramo nella sua analisi tenne conto ugualmente dei telegiornali, senonché il libro fu tolto dalla circolazione, poco dopo la sua uscita, dallo stesso editore Guaraldi, in seguito a non meglio identificate pressioni ricevute, che evidentemente dovettero essere molto convincenti.

Va ricordato d'altra parte che la d'Eramo poté sperimentare anche in positivo l'enorme potere comunicativo della televisione. Nel 1979 il suo primo romanzo, Deviazione, era comparso da qualche mese in libreria e aveva già ricevuto un gran numero di recensioni altamente positive da parte di grossi nomi sui più importanti quotidiani e settimanali, ma le vendite seguivano un corso normale; a quel punto la scrittrice fu invitata in una trasmissione televisiva di nuovo genere per l'Italia, il primo talk show condotto da Maurizio Costanzo, Caffe Grand'Italia (era forse la prima volta che uno scrittore andava in tv a parlare di un suo libro, al di fuori delle rubriche dedicate esclusivamente alla letteratura e relegate in terza serata). Di colpo Deviazione divenne un best-seller con 200000 copie vendute, destinate a moltiplicarsi fino a molte centinaia di migliaia nelle edizioni tascabili e dei club del libro. Fu probabilmente il primo caso del genere in Italia.

In questo quadro di esperienze è maturato il romanzo Nucleo Zero, il secondo della d'Eramo, che è particolarmente interessante per il nostro tema. Il libro è del 198I, e racconta una storia di terroristi che si svolge alla fine del 1980: Luce d'Eramo è stata la prima in Italia ad affrontare narrativamente il tema del terrorismo, interessandosi non solo a come si diventa terroristi, ma a come si diventa vivendo da terroristi; e lo ha fatto a caldo, in un'epoca in cui uno scrittore che considerasse queste persone come esseri umani da capire veniva automaticamente sospettato di voler giustificare i metodi terroristici e doveva giustificarsi a sua volta. Ed è rimasta la sola, a mia conoscenza, a raccontare il terrorismo dal punto di vista del rapporto con i mass media.

Il terrorismo di cui si parla qui non è naturalmente quello dei nostri giorni, ma quello che c'era in Italia in quel periodo (siamo nei cosiddetti 
anni di piombo); in particolare si parla dei gruppi clandestini di estrema sinistra. Luce d'Eramo aveva seguito gli sviluppi della lotta armata fin dagli esordi, interessandosi al linguaggio con cui venivano rivendicate le azioni e contemporaneamente al modo in cui ne davano notizia i media. Quando esce Nucleo Zero, in Italia la lotta armata, dopo aver toccato il culmine nel $1978 \mathrm{col}$ sequestro Moro, stava iniziando il suo declino. Gli attentati omicidi a dire il vero si moltiplicavano, ma dopo Moro non riuscivano più a colpire così in alto, e quindi ripiegavano su obiettivi più facilmente raggiungibili, soprattutto giudici e professori universitari consulenti del governo. L'opinione pubblica era sempre più satura di questi ammazzamenti che colpivano persone sconosciute ai più, e di cui si faticava a credere che fossero tanto nemici del popolo da meritare la morte; i lunghissimi documenti politici e i comunicati che rivendicavano gli attentati, ritualmente bollati in televisione con l'aggettivo "deliranti» e/o «farneticanti», non venivano più letti da nessuno, se non da qualche politologo e dalle questure. Inoltre, e soprattutto, nelle file degli arrestati si moltiplicavano i pentiti che denunciavano i compagni e provocavano nuovi arresti. $\grave{E}$ in questa situazione insieme di proliferazione e di crisi strisciante della lotta armata che s'inserisce la vicenda di Nucleo Zero. Il gruppo protagonista del romanzo, il Nucleo Zero appunto, non è ricalcato sul modello delle formazioni storiche che agivano allora, come le Brigate Rosse, Prima Linea, i Nuclei Armati Proletari, ma è un gruppo immaginario dialetticamente contrapposto a questi, per cui il racconto pone questioni che sono di grande attualità ancor oggi, quando il terrorismo è tornato alla ribalta in modi inimmaginabili rispetto agli anni Ottanta.

In Nucleo Zero il terrorismo è visto come effetto dell'adozione, da parte di gruppi eversivi, di una mentalità massmediatica, nel senso che ogni atto terroristico mira a imporsi innanzitutto come atto di comunicazione, che tiene esplicitamente conto, nella sua progettazione, della reazione dei mass media, e presume di potersene servire per costringerli a diffondere il messaggio rivoluzionario.

Il Nucleo Zero delle Bande comuniste è un gruppo che si dedica esclusivamente all'autofinanziamento, organizzando rapine con tecniche inconsuete e ineccepibili, curate in ogni dettaglio, con l'obiettivo di ridurre al minimo i rischi di essere scoperti. Sono rapine che non vengono mai rivendicate come espropri proletari e passano per reati comuni. Il fondatore di Nucleo Zero, Giovanni Dettore, già professore di filosofia, e la sua compagna Lorenza, un tempo giornalista, provengono dalle file di una formazione tradizionale della lotta armata, le Colonne Rosse, che assomigliano molto alle vere Brigate Rosse. Il motivo per cui se ne sono 
staccati lo ricorda lo stesso Dettore ai suoi compagni che vorrebbero cambiare strategia, nel corso di una riunione drammatica in cui critica gli ex compagni:

Tutto per l'illusione di trovare una scorciatoia alla rivoluzione. E con che cosa? Per mezzo dei mass media. Con le stesse mammelle da cui le masse succhiano il latte del loro assoggettamento. [...] Appena eseguita, ogni azione esemplare diventa scontata; quasi diminuita, agli occhi dell'opinione pubblica, dal suo essere fattibile. E ce ne vuole una più esemplare ancora. Un'escalation. [...] Così la lotta armata è degenerata in terrorismo. Colonne \& C. ridotte a sbalordire i borghesi. E siccome ciò che più titilla i benpensanti è il delitto, Colonne \& Company si sono risolte in comuni associazioni a delinquere. E voi vorreste rammanicarvi con loro, con queste veteroformazioni fuorilegge ormai note a tutte le polizie e a tutti i servizi segreti del mondo [...] Nel farsi vive ogni tanto, con qualche sparata (sempre più letterale) [...] sembrano dire ai propri cacciatori: cercateci, siamo qui, siamo là. In un nascondino patetico. Che a quanto pare vi attira. [...] — Perché ti fermi a mezza strada? - gli aveva risposto Stefano Brandi - Se ci rifletti, il rivoluzionario più clandestino, l'imprendibile per eccellenza, è quello che non esiste. E noi siamo su questa via. Per non farci stanare dai mass media, non firmiamo le nostre azioni. [...] Risultato: non possiamo compiere azioni politiche. (NZ, pp. I24-I25.)

Il contrasto è lacerante: servirsi dei mass media porta a un'escalation del crimine che allontana dai propri scopi e disgusta quelle masse stesse che si volevano raggiungere; il filtro dei media trasforma a priori i militanti in mostri; aspirate dalla tv, le loro azioni vengono restituite cambiate di segno, il loro significato irreparabilmente alterato rispetto a quello che gli attribuisce chi le compie. D'altra parte la clandestinità e il mutismo portano egualmente a una cancellazione della propria ragion d'essere, e alla non esistenza di fatto sul piano politico.

Nel romanzo Dettore persiste nella propria strategia di silenzio assoluto, ma viene messo in minoranza dai compagni e questo gli costa sul piano personale la rottura con Lorenza e l'isolamento. "Tu hai insinuato che io mitizzo la clandestinità operativa», dice Dettore al suo ex allievo Stefano Brandi. "Trovami un'altra difesa dalla macina dei mass media, dai guasti incommensurabili del meccanismo pubblicitario, e io starò con te.» (NZ, p. 193.) Ma gli altri del Nucleo Zero non lo ascoltano e decidono di collaborare con le Colonne Rosse, per realizzare quella che agli occhi di Dettore è appunto un'escalation per pubblicizzare se stessi agli occhi delle masse, che li porterà a farsi macinare dai media. Sequestreranno il padrone della massima industria italiana, la Rotat, e in cambio del suo rilascio chiederanno una serie di misure economiche e giuridiche a favore degli operai.

$\mathrm{Nel}$ corso di questo sequestro il problema ritorna in scontri assai aspri tra i due gruppi eversivi alleati. La strategia di comunicazione proposta dal 
giovane Stefano Brandi, che sta prendendo la leadership del Nucleo Zero al posto di Dettore, scardina completamente i binari convenzionali, che sono: attentato - rivendicazione - comunicati di analisi politica - trattative col potere - braccio di ferro per ottenere un riconoscimento da parte dello Stato, poi, qualunque sia l'esito, nuovi comunicati, liquidati dai commentatori in giudizi sommari e destinati a non arrivare mai alle masse (e, se ci arrivassero, incomprensibili).

Secondo Brandi, invece, al nemico di classe

bisogna portargli la guerriglia nel cervello, dissestarne gli inquadramenti mentali, sorprenderne le categorie di giudizio. Perciò, facciamo tutti insieme i sequestri politici senza firme e senza sangue, cioè esattamente come noialtri delle bande facciamo le rapine. [...] Un pezzo grosso è rapito ma non si sa da chi. [...] L'establishment salta come un picchio, i mass media hanno la loro brava crisi isterica, e noi zitti. Quando le emittenti radiotelevisive e i giornali boccheggianti hanno mollato l'osso e parlano d'altro, noi rilasciamo il nostro uomo lindo e pulito, rimesso a nuovo con gentili risciacquature del cervello, che ricompare indesiderato tra i suoi cari. Un fu Mattia Pascal di pirandelliana memoria. [...] O abbiamo scelto la lotta armata, e allora fatti, non parole; o non siamo capaci di rinunciare al dialogo e allora restiamo nella controinformazione. O l'uno o l'altro. Se scegliamo i fatti, dobbiamo credere nella loro espressività e perciò dargliela in pieno. Ma fifti fifti, rincalzare le azioni con le parole, significa azzopparle tutte e due. (NZ, pp. 199-200.)

Ma la strategia innovativa del Nucleo Zero non sfonda. Il sequestro si svolge secondo uno schema parzialmente diverso da quello abituale, frutto del compromesso tra le due organizzazioni. Al di là dell'esito immediato del sequestro (cedimento della Rotat o esecuzione del prigioniero), la posta in gioco è il messaggio che passerà. Di questo sono convinti entrambi i gruppi, ma sono divisi sul senso che le masse attribuiranno alle loro azioni. Dunque la discussione dei terroristi è in primo luogo in termini di risultati nella percezione del pubblico: questo è considerato il fattore politico decisivo, ed è questo che rende così moderno e attuale questo romanzo.

In ultima analisi, quelli delle Colonne Rosse sono convinti che i proletari conservano una percezione diversa, autonoma da ciò che il potere vuol far loro credere, mentre i nucleisti sono convinti che proprio le classi subalterne sono le più indifese di fronte ai messaggi dei media, in primo luogo della televisione.

Conclusosi drammaticamente il sequestro con la condanna a morte dell'ostaggio, la vicenda precipita: la polizia è sulle tracce di Lorenza. A catena, tra morti e arresti, il gruppo è rapidamente costretto a disperdersi. Nell'ultima scena del romanzo, l'unico superstite del Nucleo Zero, Stefano, assiste in tv agli ultimi atti della dissoluzione del gruppo alleato: la morte di Toson, l'arresto della sua compagna. L'ultima parola spetta al 
redattore del telegiornale, anche se noi lettori sappiamo che il giovane terrorista inchiodato davanti al teleschermo sta già organizzando un nuovo gruppo che faccia tesoro dell'esperienza precedente, ancora più coperto, ancora meno riconoscibile, ancora più refrattario alla tentazione di comunicare. Sul piano narrativo, uno spiraglio resta aperto; non sapremo mai se il tentativo di uscire dai binari obbligati e di inventare una guerriglia sul piano per così dire semiologico avrà in qualche modo uno sbocco; se le mute Bande comuniste si siano poi estinte nel silenzio o stiano ancora faticosamente moltiplicandosi in un'incubazione infinita. Sul piano della realtà, si direbbe che non hanno trovato seguaci, considerando come i nuovi terrorismi continuino a rapportarsi frontalmente con le televisioni e persino con internet, a quanto è dato di giudicare.

L'impressione che la lettura del romanzo lascia dei primi anni Ottanta è quella di una società in cui i canali dell'informazione di massa, in primo luogo la tv, sono saldamente nelle mani delle classi dominanti; restano nella stampa piccoli spazi di controinformazione e di dissenso, influenti solo marginalmente, e sempre meno visibili sotto il sole televisivo. Tentare di piegare i media a fare da megafono a un piccolo movimento antagonista, con azioni terroristiche e scontri frontali dichiarati, è perdente e autodistruttivo sul terreno stesso della comunicazione. Ciò vale in primo luogo per la televisione: non perché il grosso della stampa si muova molto diversamente, ma perché il potere di penetrazione della televisione, la sua presa diretta sugli eventi sono incomparabilmente maggiori, e perché incomparabilmente maggiore è il grado di compattezza interna e di omologazione dei programmi televisivi.

Questo è il motivo per cui in Nucleo Zero i telegiornali hanno un ruolo essenziale nella narrazione. Perché sono loro che mediano i gesti dei protagonisti agli occhi dei destinatari, gli uomini e le donne che potrebbero volersi ribellare a un destino di passività. E allora per esempio nella scena finale di cui parlavamo, vediamo il linguaggio televisivo all'opera: la morte del poliziotto è annunciata come la tragica fine di un giovane di 24 anni, colpito da una terrorista; «il suo cuore ha cessato di battere» dopo una lunga lotta, nonostante tutti gli sforzi per salvarlo; vediamo scorrere sullo schermo le sue foto da bambino, poi allegro con la fidanzata, seguono i messaggi di cordoglio delle autorità. Segue poi la secca notizia di un uomo trovato morto su un marciapiede, la testa spaccata. È un terrorista da tempo ricercato. "Una foto segnaletica della faccia di Toson campeggia sul video - occhi diacci, labbra sigillat.» Il teleredattore afferma che il criminale tentando di fuggire è precipitato. Le immagini mostrano il cornicione, il frammento staccato sull'asfalto. Montaggio delle imma- 
gini e commento sonoro si intrecciano nella comunicazione delle notizie creando effetti di realtà al posto delle ipotesi, effetti emotivi di attrazione e di repulsione, indirizzando implicitamente le opinioni nell'apparente distacco e minuziosa obiettività.

Dal canto loro i terroristi del Nucleo Zero, per minimizzare i rischi di essere scoperti, hanno ridotto all'osso le comunicazioni tra loro. Anche privatamente, non possono scriversi e telefonarsi; salvo eccezioni rigorosamente preventivate, non devono frequentarsi, se non lo giustifica il loro lavoro di copertura ufficiale.

Così nel romanzo si intrecciano due codici informativi non comunicanti: quello pubblico dei telegiornali e quello segreto dei terroristi, che nelle chiamate telefoniche per evitare intercettazioni hanno adottato un codice di squilli numerati. Si tratta di un codice scheletrico, che non può dar risposte a tutte le loro domande e ai loro dubbi e non dà spazio al loro bisogno di esprimersi; per cui i terroristi soffrono sempre per l'insufficienza di comunicazione tra loro. Un argomento che ho esaminato altrove (Ambrosino, 200I, pp. 2I4-2I5) è come questa sfiducia estrema nella parola, e questa rinuncia alla comunicazione finisca alla lunga per minarli nella loro ragion d'essere. Qui vorrei solo notare il loro contraddittorio rapporto con la televisione: i terroristi sono sempre attentissimi agli orari del telegiornale e spesso è proprio dal telegiornale che apprendono le notizie che li riguardano o riguardano i compagni, vedono raccontare le proprie azioni in retrospettiva, scoprono a volte errori e disattenzioni nella loro messe in scena, si riconoscono nei loro travestimenti col cuore in gola. Insomma se rinunciano al palcoscenico televisivo sono i primi a non poter fare a meno del suo specchio, per quanto deformante lo sentano. Non solo perché consente loro di sapere se e come li vedono gli altri, ma per avere notizie di ciò che sta accadendo ai compagni, per informazioni vitali, per capire se la polizia è sulle loro tracce. Nel corso della narrazione sono sempre i telegiornali, spesso ascoltati nei bar, tra la folla, mescolati agli altri avventori, che segnano le svolte.

Ed è lì che i banditi misurano l'effetto della trasmissione, gli umori del pubblico, l'immenso potere di attrazione del mezzo televisivo sulle cosiddette masse. C'è l'episodio dell'apprendista meccanico che lavorava nell'officina di Dettore.

Una mattina era arrivato eccitatissimo annunciando che quella sera lui sarebbe apparso in tivù, terza rete tra le 2 I.I5 e le $2 \mathrm{I} .30$ [...]. Dettore aveva acceso il teleschermo e, veramente, per un attimo la figura dell'apprendista sedicenne era apparsa a un semaforo mentre si riprendeva l'inaugurazione della metropolitana. L'indomani l'apprendista era venuto al lavoro col viso raggiante, - M'avete visto? - andava chiedendo agli operai, - Ero in tivù - sorrideva. (NZ, p. 277.) 
Non c'è ancora, in questo libro del I98I, e non potrebbe esserci, il dispiegamento in tutte le sue forme della società dello spettacolo, il dilagare della televisione commerciale, l'avvento dei reality in cui ciascuno si affretta a portare in tv la propria vita più intima e si ritrova a vivere televisivamente anche in privato. Ma ce ne sono già, in questo garzone felice di essere apparso un attimo, tutte le premesse. $\mathrm{E}$ in altre pagine relative al sequestro Perrino (NZ, pp. 244-245) c'è anticipata la capacità della televisione di neutralizzare anche le occasioni degli antagonisti, ad esempio il discorso satirico, se gli strati sociali dominanti, che ne dovrebbero essere il bersaglio, saranno abbastanza lungimiranti da servirsene come valvola di sfogo per gli scontenti e come conferma di un'immagine di società aperta, pluralistica, liberale.

Quello che rende tragico il destino del Nucleo Zero, è che persone capaci di spogliarsi di beni, vita, storia personale (sono infatti disposti anche a perdere la propria identità e i propri connotati) per inseguire il sogno di una società di esseri umani finalmente alla pari, siano poi indotte a privarsi della parola, ossia di ciò che per loro è più vitale, la comunicazione coi propri simili. Ai loro occhi, al di fuori di un mutismo sovversivo, non c'è modo di sottrarsi all'imbuto mediatico di una democrazia ingannevole, una fabbrica di consenso che non ci appartiene, che funziona a senso unico e che tutti finiamo per alimentare, qualunque cosa diciamo e quali che siano le nostre intenzioni.

Fin qui abbiamo esaminato il ruolo della tv all'interno del romanzo. Ma dato che Nucleo Zero nel 1984 è stato trasposto in un film per la televisione, prodotto da RAI e da FRI, con sceneggiatura di Ugo Pirro e regia di Carlo Lizzani (entrambi cineasti di valore e uomini di sinistra), abbiamo l'opportunità di verificare anche il rapporto della tv reale con un'opera letteraria in un caso preciso, confrontando il romanzo con il suo adattamento televisivo.

Sul piccolo schermo la vicenda è stata, più che modificata, trasformata. Non si tratta dello sfoltimento necessario per comprimere in poco tempo una vicenda complessa: il tempo non è stato lesinato, perché al soggetto sono state dedicate tre ore di trasmissione, da dividere in tre puntate. Senza entrare nei particolari, constatiamo che le azioni spettacolari del gruppo sono conservate e rese con grande tensione e suspence; il sequestro dell'industriale, cioè l'episodio fondamentale del romanzo, è stato praticamente eliminato; dilatando invece uno spunto del romanzo, la dinamica della sconfitta dei terroristi viene riscritta in modo completamente capovolto. Segue poi il processo ai terroristi, che nel romanzo non esiste, e che qui 
prende molto spazio, illustrando il pericolo corso dalla società e il faticoso ristabilimento dell'ordine. In questo processo l'avvocato Di Biasio e suo figlio (divenuto avvocato anche lui) si assumono la difesa dei terroristi. Al centro della narrazione filmica c'è un problema morale del tutto estraneo al romanzo: il dramma psicologico di un padre borghese che non se la sente di denunciare il figlio ex terrorista, del figlio terrorista ravveduto che non se la sente di denunciarsi, ma si sente in colpa verso gli ex compagni, ragion per cui si trovano entrambi a disagio in una posizione di ambiguità. Chi è costretto al mutismo, qui, sono soprattutto questi due insospettabili, che portano con sé il loro pesante segreto.

Salta agli occhi che del rapporto del terrorismo coi mass media e con la televisione, che è al cuore del romanzo, non è rimasta nessuna traccia.

Personalmente ricordo che Luce d'Eramo, che aveva incautamente ceduto i diritti di adattamento prima di vedere la sceneggiatura, con piena fiducia nel regista Lizzani, rimase sbalordita. Tentò in tutti i modi di far cambiare la sceneggiatura, ma le fu spiegato che non era possibile: impensabile far produrre per la tv la storia com'era, e poi ormai non si poteva più tornare indietro. Ma a parere degli adattatori - benché non condiviso dall'autrice - il senso fondamentale del romanzo non era andato perduto, anzi era stato esplicitato. La storia dei rapporti tra letteratura e televisione è fatta anche di questo tipo di travisamenti.

\section{Bibliografia}

Eramo Luce (d'), Cruciverba politico. Come funziona in Italia la strategia della diversione, Firenze, Guaraldi, 1974.

Eramo Luce (d'), Nucleo Zero, Milano, Mondadori, i98I. [NZ]

\section{Su Nucleo Zero:}

Bertini F., «Luce d'Eramo, Nucleo Zero», Il Ponte, Firenze, 3I luglio 1982. Bevilacqua Alberto, "Come l'intrigo clandestino diventa intrigo di romanzo", Il Corriere della Sera, Milano, I6 novembre i98I.

Camon Ferdinando, "Nel nucleo del terrore», Il Giorno, Milano, 4 nov. I98I.

Ferretti Gian Carlo, «Terroristi romanzati», Rinascita, Roma, 23 ottobre I98I.

GiUdici Giovanni, «Battaglia navale con il terrorista», l'Unità, Roma, 29 ottobre I98I. 
Lombardi O., «Luce d'Eramo - Nucleo Zero», Nuova Antologia, Firenze, giugno 1982.

Marabini C., "Il primo romanzo sul terrorismo - Quel mondo è così», La Nazione, Firenze, I9 gennaio 1982.

Mauro Walter, "Il romanzo per penetrare nel covo», Il Popolo, Roma, 27 ottobre 198I.

Mieli P., "Un romanzo sul terrorismo - Le $\mathrm{Br}$ tinte di giallo», L'Espresso, Roma, I9 aprile I98I.

Pappalardo La Rosa Franco, «Le radici della violenza», L'Umanità, Roma, Io novembre I98I.

Porzio D., «Nucleo Zero di Luce d'Eramo», Panorama, Milano, 7 dic. I98I.

Scurani A., "Nucleo Zero di Luce d'Eramo», Letture, Milano, gennaio I982.

Starnone Domenico, «Il nucleo invisibile del terrore. L'ultimo romanzo di Luce d'Eramo, un dibattito a Roma», Il Manifesto, Roma, 25 marzo I982.

\section{Sulla narrativa di Luce d'Eramo:}

Ambrosino Daniella, "Temi, strutture e linguaggio nei romanzi di Luce d'Eramo", Linguistica e letteratura, XXVI, 200I, pp. 195-25I.

Eramo Marco (d'), Vanzan Piersandro [a cura di], «Speciale Luce d'Eramo. Scritti di Marco d'Eramo, Piersandro Vanzan, Daniella Ambrosino, Corinne Lucas-Fiorato, Paola Ferro, [con un inedito di Luce d'Eramo]", Prospettiva Persona, XXII, 2003, pp. 55-74.

Ferretti Gian Carlo, Il best seller all'italiana. Fortune e formule del romanzo di qualità, Roma-Bari, Laterza, 1983.

LuCas Corinne, «Des colonnes d'Hercule à Nnoberavez : l'art du déplacement dans l'œuvre de Luce d'Eramo», Mélanges offerts à Pierre Laroche, Études réunies par Denis Ferraris et Danièle Valin, Chroniques italiennes, n. 69-70, 2002, pp. II3-I27. 